\title{
Human Factors and Ergonomics Society $50^{\text {th }}$ Annual Meeting
}

\author{
Tuan Q. Tran \\ Kimberly R. Raddatz
}

October 2006

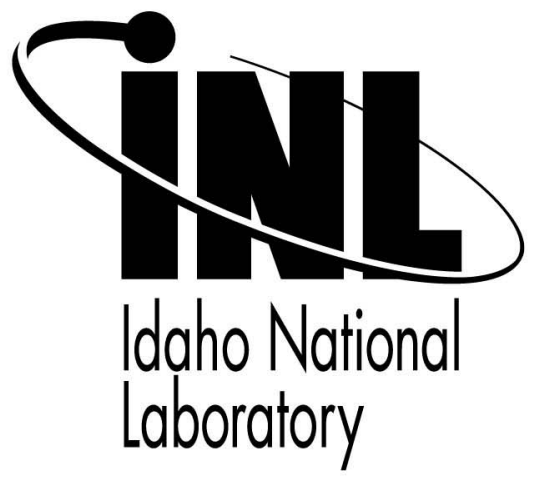

This is a preprint of a paper intended for publication in a journal or proceedings. Since changes may be made before publication, this preprint should not be cited or reproduced without permission of the author. This document was prepared as an account of work sponsored by an agency of the United States Government. Neither the United States Government nor any agency thereof, or any of their employees, makes any warranty, expressed or implied, or assumes any legal liability or responsibility for any third party's use, or the results of such use, of any information, apparatus, product or process disclosed in this report, or represents that its use by such third party would not infringe privately owned rights. The views expressed in this paper are not necessarily those of the United States Government or the sponsoring agency. 


\title{
The Effects of Transient Emotional State and Workload on Size Scaling in Perspective Displays
}

\author{
Tuan Q. Tran \\ Idaho National Laboratory \\ Idaho Falls, ID
}

\author{
Kimberly R. Raddatz \\ Kansas State University \\ Manhattan, KS
}

\begin{abstract}
Previous research has been devoted to the study of perceptual (e.g., number of depth cues) and cognitive (e.g., instructional set) factors that influence veridical size perception in perspective displays. However, considering that perspective displays have utility in high workload environments that often induce high arousal (e.g., aircraft cockpits), the present study sought to examine the effect of observers' emotional state on the ability to perceive and judge veridical size. Within a dual-task paradigm, observers' ability to make accurate size judgments was examined under conditions of induced emotional state (positive, negative, neutral) and high and low workload. Results showed that participants in both positive and negative induced emotional states were slower to make accurate size judgments than those not under induced emotional arousal. Results suggest that emotional state is an important factor that influences visual performance on perspective displays and is worthy of further study.
\end{abstract}

\section{INTRODUCTION}

Significant effort has been devoted to enhancing the experience and productivity of two-dimensional (2-D) perspective displays. Most of this effort has been focused on exploring the impact of specific display design factors (e.g., number and type of depth cues, magnification, etc.) on observers' sensory, cognitive, and physical response capacities for making spatial judgments such as object size scaling. The ability to perceive the distal (objective) size of objects despite the fact that proximal (retinal) size changes with viewing distance (i.e., size constancy) is a direct measure of veridical perception in perspective displays. Past research has shown that under certain conditions size constancy maintains in 2-D perspective arrays (e.g., Uhlarik, Pringle, Jordan, \& Misceo, 1980).

Factors influencing size constancy in 2-D displays are many and span both the perceptual and cognitive domains. For example, certain pictorial depth cue combinations (e.g., Bruno \& Cutting, 1988) and instructional sets (e.g., Carlson, 1960) produce judgments that deviate from size constancy. However, less is understood about the impact of observers' transient emotional state on the ability perceive veridical size in 2-D displays.

An individual's emotional state has been shown to influence how individuals judge and process information (e.g., Clore, Schwarz, \& Conway, 1994). Specifically, researchers have proposed that emotional state may lower the level at which individuals focus on information (Clore \& Gasper,
2001). According to the level of focus hypothesis (Clore, Gasper, \& Garvin, 2001), happy moods may compel individuals to focus on information at a global level, focusing on the stimulus as a unitary whole whereas sad moods may compel individuals to think about information at a more local level, focusing on the details or component properties of the stimulus.

The impact of emotional state on size scaling in perspective displays has important implications, considering that these displays often must support judgment and decision-making in environments where misperceptions have severe consequences. For example, terrain-enhanced primary flight displays present perspective views of terrain that are designed to facilitate pilots' avoidance of controlled flight into terrain (CFIT) accidents. Cockpit displays of traffic information (CDTIs) shown in perspective are designed to enhance pilots' ability to avoid traffic collisions. In these examples, the accurate scaling of size across projected depth is the precursor to the spatial judgments that must be made to maintain safety. Further, these environments can also induce high arousal and workload, which may, in turn, influence the pilot's emotional state. Therefore, it is important to understand how spatial judgments in perspective displays, specifically size scaling, are impacted by observers' emotional state and how emotional state may interact with high workload. 


\section{Emotion \& Working Memory (WM)}

The interaction between workload and emotion has been well recognized within cognitive literature and is based on the fundamental assumption that humans have limited attentional resources to devote to tasks. According to Kahneman (1973), capacity available for information processing can be modulated by emotional arousal. This cognitive capacity-reduction hypothesis asserts that emotional arousal (both positive and negative) can drain attentional resources, resulting in the decreased effectiveness of WM processes required for highercognitive functioning (e.g., decision-making, problem-solving). Further, the degree of capacity reduction and its impact on performance is positively related to the degree of emotional arousal (Hasher, Zacks, Rose, \& Doren, 1985).

Given that size scaling requires effortful processing (e.g., Carlson, 1960) and a demand on attentional resources in WM, the present study examined factors that may compete for (e.g., emotions) or further deplete (e.g., workload) this limited resource capacity. Thus, under low workload conditions, it is hypothesized that size judgments will be unaffected by emotional arousal. Even with the depletion of attentional resources by emotional arousal, individuals under low workload should still possess sufficient resources to support veridical size judgments. However, size judgments made under heavy workload will require more attentional resources, some of which will have already been drained by emotional arousal. Thus, size judgments under high workload and high arousal should be less veridical compared to those made under neutral arousal.

\section{METHOD}

\section{Participants}

One-hundred and six native English speakers were randomly assigned to one of three emotional states (negative, neutral, and positive). Data from twenty-three participants (i.e., four in the negative condition, nine in the neutral condition, ten in the positive condition) were discarded due to extremely high error rates (over $50 \%$ error) in the size judgment task.

\section{Materials}

Emotion was manipulated with emotionallyladen film clips. Participants in the happy condition watched a segment from "When Harry Met Sally" depicting two adults discussing whether women can fake sexual excitement. Participants in the sad condition watched a segment from "The Champ" portraying a boy's tearful reaction to his father's fatal injuries in a boxing match. Participants in the neutral condition watched a segment from "Shapes" displaying colorful geometric bars simulating a screen saver. All film clips ran approximately $2 \frac{1}{2}$ minutes and were taken from Gross and Levenson (1995).

The Brief Mood Introspection Scale (BMIS; Niedenthal \& Setterlund, 1994) was used to measure participants' sad and happy emotional states initially and at various times after induction. The BMIS lists 16 affect adjectives (e.g., lively, happy, sad, tired, content, active, etc.) embedded in the sentence: "I presently feel ." Participants indicated the degree to which they are presently feeling each statement on a 5-point Likert scale.

Size Judgment Stimuli. The perspective displays were generated using commercially available terrain generating software and provided a static view of two red blocks resting on a receding flat perspective terrain surface. The relative distance (in depth) between the two blocks varied from $0,3,6$, or 9 units (see Figure 1) with 0 units representing blocks presented side-by-side. Participants were asked to judge whether the blocks had the "same" or "different" distal widths (Note: Distal heights and depths of the blocks were distally equal and invariant).

They were given phenomenal, or "first-look," instructions asking them to make the width comparisons based on how the distal width of each block "appeared" to them, which may not have necessarily corresponded with the actual distal width of the block (Carlson, 1960). Participants were told to respond as accurately and rapidly as possible by pressing the appropriate response key. Participants did not receive feedback regarding their size judgments.

The size judgment task was comprised of two levels of difficulty. "Difficult" trials were those trials in which the distal size ratio between the two 
blocks was $2: 3$ or $2: 1$ and the distance difference between the two blocks was either 6 or 9 units. "Easy" trials were those trials in which the distal size ratio was $1: 1$ or $2: 1$ and the distance difference between the two blocks was either 0 or 3 units.

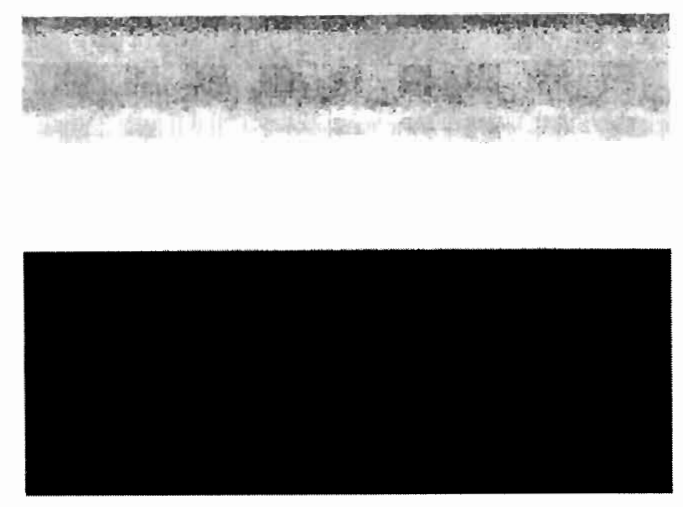

Figure 1. Example of stimuli used in size judgment task.

Workload Manipulation. The size judgment task was embedded within a Modified Sternberg Task (MST; Oberauer, 2001) in which participants memorized a list of words and then responded whether or not a probe word was in the memorized list. Words were closely matched on frequency, length, syllables, valence and arousal ratings.

The word list was presented in two lines with the number of words in each line comprising the workload manipulation. Specifically, a memory set size of 1 word per line comprised the low workload condition while a memory set size of 3 words per line comprised the high workload condition. Both memory lines were comprised of either 1 word or 3 words each.

Picture Task. The picture task was presented at the end of each MST trial to reduce the build-up of proactive interference from previous trials and to further induce and maintain participants' emotion arousal originally induced by the film clip. Regardless of emotional arousal conditions, all pictures in MST Block 1 were neutral (e.g., cup, table, chair, etc.) to reduce the possibility that the picture task would inadvertently induce positive or negative emotion before the actual emotion induction via the film clip.

Picture valence was congruent with each participant's induced emotion state (e.g., "happy" participants saw only positive pictures, etc.)

Participants were asked to rate the degree of valence (positive/negative) of each picture on a seven point scale.

E-prime experimental software was used to control the presentation of the MST, size judgment, and picture stimuli, and to collect response data.

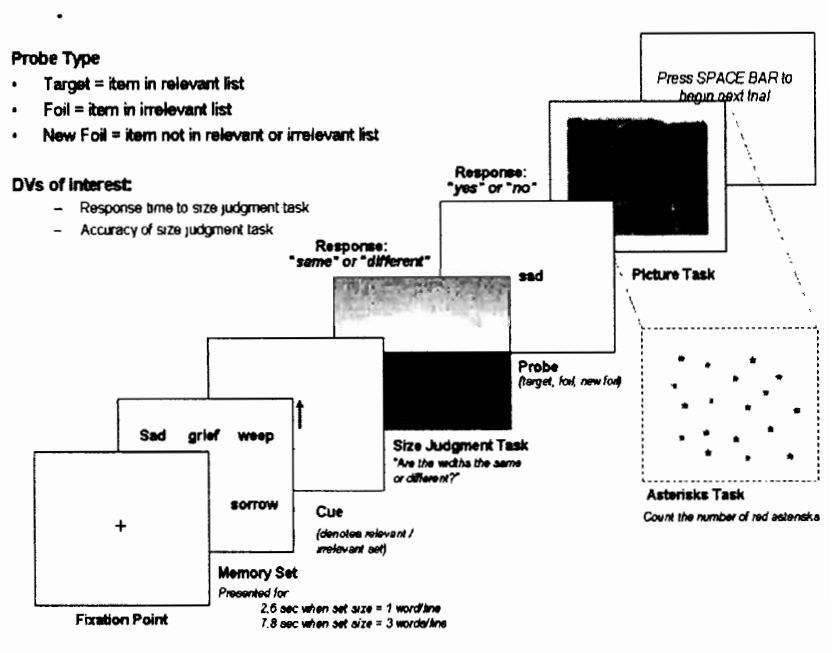

Figure 2. Schematic of each trial of the Modified Sternberg Task (MST). Note that the size judgment task was embedded in a larger experiment.

\section{Design and Procedure}

The experiment consisted of a 3 (Emotional State: negative, neutral, and positive) $\times 2$ (Memory Load: set size $=1$ word, set size $=3$ words) $\times 2$ (Size Judgment Difficulty Level: easy, difficult) mixed design with Emotional State as the between-subjects variable.

Each trial was comprised of the MST embedded with the size judgment and picture tasks. Each trial began with the presentation of a fixation point (1000ms), followed by the memory set of two lines (Figure2). Participants were told to memorize both lines. After a short duration ( $2.6 \mathrm{sec}$ for set size $=1$ word and $7.8 \mathrm{sec}$ for set size $=3$ words), the memory set was replaced by an arrow pointing to the line on which participants' memory would be tested. The arrow was presented for $800 \mathrm{~ms}$, followed immediately by the size judgment stimulus. Participants were to press the " 1 " key if 
the block widths were the same and " 2 " if they differed. The size judgment task remained on the screen until participants made a response or until $2500 \mathrm{~ms}$ expired. Probe word presentation immediately followed the size judgment. Participants were to quickly and accurately respond whether or not the probe word was in the relevant memory line, responding "yes" with the " 1 " key and "no" with the " 2 " key, followed immediately by visual response feedback. Finally, participants saw either a picture or an array of asterisks printed in black, blue, green, and red ink. They rated the valence of the picture or counted the number of red asterisks. Responses were made using the keypad.

Participants were tested individually in groups of 1,2 , or 3 on separate computer terminals. They were given 12 practice trials to familiarize themselves with the MST, the size judgment task, and the picture/search task. Participants then performed an additional 36 trials in Block 1 that served as additional practice trials, although participants thought the experiment had already begun. After watching the film, participants completed the 24 critical trials in both Blocks 2 and 3 for a total of 48 critical trials. Figure 3 illustrates the entire procedure of the experiment.

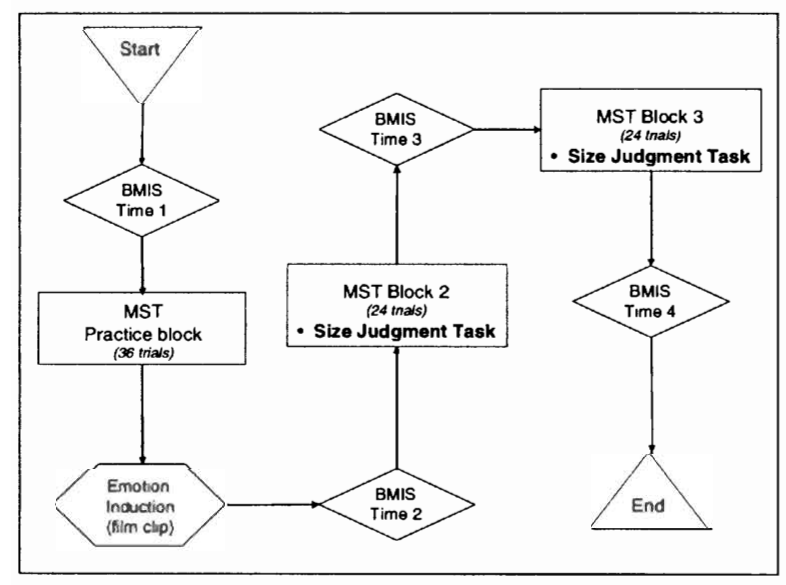

Figure 3. Diagram depicting the procedure of the experiment.

\section{RESULTS AND DISCUSSIONS}

All discussed results are significant at the .05 level unless otherwise stated.

\section{Emotion Assessment Scores}

The BMIS scores were calculated by summing four scores for sad adjectives (sad, gloom, drowsy, and tired) and five scores for happy adjectives (happy, content, lively, peppy, and active) to generate sad and happy BMIS scores (Niedenthal \& Setterlund, 1994).

One-way analysis of variance (ANOVA) was used to assess the effectiveness of the emotion manipulation. Participants did not differ in their emotional state at Time 1 . At Time 2 , immediately following the video clip, participants in the sad condition exhibited higher sad BMIS scores compared to happy participants, $F(2,80)=8.52$, while participants in the happy condition exhibited higher happy BMIS scores compared to sad participants, $F(2,80)=3.91$. However, differences in sad and happy BMIS ratings across emotional conditions at Times 3 and 4 failed to reach significance. Thus, response time (RT) and error rate analysis will only be discussed for Block 2 , during which the emotion manipulation held.

\section{Judgment Times and Error Rates}

Only RTs for correct size judgments were analyzed. RTs greater than two standard deviations beyond each participant's overall mean were replaced with their respective outer fences.

RTs and error rates were submitted to two separate 3 (Emotional State: negative, neutral, positive) $\times 2$ (Workload: memory set size $=1$, memory set size $=3$ ) x 2 (Size Judgment Task Difficulty: easy, difficult) mixed-design ANOVA, with Emotional State as the between-subjects variable.

Error rate analysis revealed only a main effect of Task Difficulty, $F(1,80)=11.03$. Not surprisingly, more errors occurred when size judgments were difficult $(M=22, S E=1.7)$ than when they were easy $(\mathrm{M}=14, \mathrm{SE}=2.2)$. $\mathrm{RT}$ analyses indicated a main effect of emotion condition, $F(2,78)=3.37$, with both "happy" (M=1451ms, SE=69) and "sad" participants ( $M=1420 \mathrm{~ms}, \mathrm{SE}=63)$ taking longer to judge size than neutral participants $(M=1222 \mathrm{~ms}$, $\mathrm{SE}=67$ ). However, post-hoc tests with Bonferroni correction revealed only a marginally significant difference between happy and neutral participants $(\mathrm{p}=.06)$. There was also a significant interaction 
between workload and task difficulty $F(1,78)=12.94$. Workload had no effect when size judgments were difficult. However, when workload was low, difficult judgments took much longer to make than easy judgments.

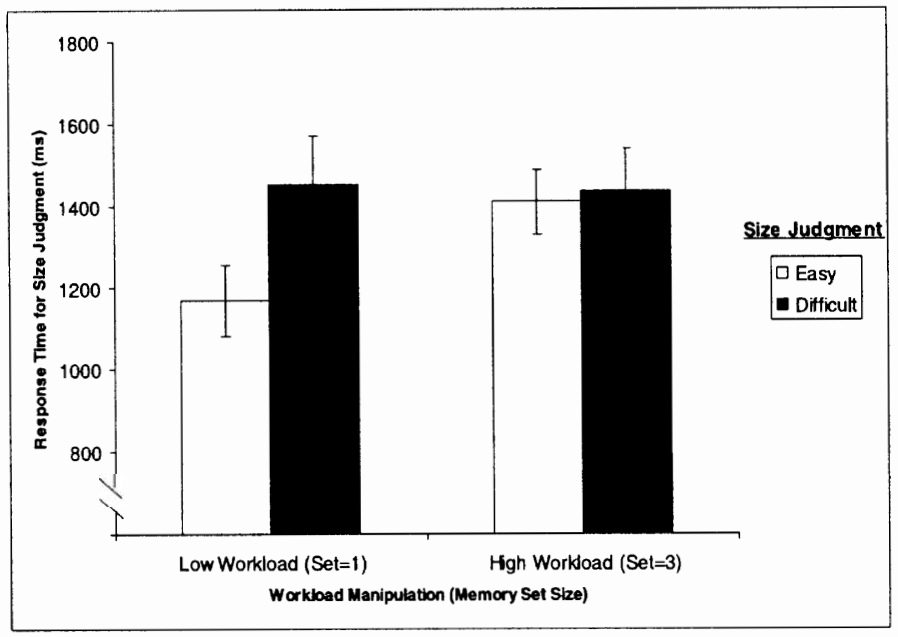

Figure 4. The interaction of workload (memory set size) and difficulty of the size judgment on participants' response times.

\section{CONCLUSIONS}

Consistent with our hypothesis, participants under emotional arousal (positive and negative) took longer to judge size compared to individuals under a neutral emotion state. This is consistent with the cognitive capacity reduction hypothesis that an individual's attentional capacity is reduced or modulated when under an emotionally arousing state (Kahneman, 1973). Also, results confirmed that both workload and task difficulty play important roles in size judgments. That is, when the workload was low, easy judgments took much less time than difficult judgments. However, when workload was high, judgments took longer, regardless of difficulty level. Not surprisingly, the more difficult the task, the more error-prone an individual becomes. In sum, these results suggest that emotional arousal does play a role in perceiving size judgments within a dual-task paradigm.

Because the size judgment task was the secondary task embedded in a larger study, judgments were made under a challenging dual-task paradigm. Future research will further examine whether participants' emotional state and workload impact size judgments as the primary task. Also, future studies will examine exactly what aspect(s) of size perception are influenced by emotional states (e.g., sensitivity or response bias).

\section{ACKNOWLEDGMENTS}

This paper was completed while the first author was a doctoral student at Kansas State University.

\section{REFERENCES}

Bruno, N. \& Cutting, J. (1988). Minimodularity and the perception of layout. Journal of Experimental Psychology: General 117(2), 161-170.

Carlson, V. (1960). Overestimation in size-constancy judgments. American Journal of Psychology, 63, 199-213.

Clore, G. L., \& Gasper, K. (2001). Feeling is believing: Some affective influences on belief. In N. Frijda, T. Manstead, \& S. Bem (Eds.), Handbook of social cognition ( $2^{\text {nd }}$ ed., pp. 323-34l). Hillsdale, $\mathrm{NJ}$ : Lawrence Erlbaum Associates, Inc.

Clore, G. L., Gasper, K., \& Garvin, E. (2001). Affect as information. In J. P. Forgas (Ed.), Handbook of affect and social cognition (pp. 121-144). Mahwah, NJ: Lawrence Erlbaum Associates, Inc.

Clore, G. L., Schwarz, N., \& Conway, M. (1994). Affective causes and consequences of social information processing. In R. S. Wyer \& T. K. Srull (Eds.), Handbook of social cognition $\left(2^{\text {nd }}\right.$ ed., Vol. 1, pp. 323-418). Hillsdale, NJ: Lawrence Erlbaum Associates, Inc.

Gross, J., \& Levenson, R. (1995). Emotion elicitation using films. Cognition and Emotion, 9, 87-108.

Hasher, L., Zacks, R. T., Rose, K. C., \& Doren, B. (1985). On mood variation and memory. Reply to Isen (1985), Ellis (1985), and Mayer and Bower (1985). Journal of Experimental Psychology: General, 114, 404-409.

Kahneman, D. (1973). Attention and effort. New Jersey: Prentice Hall.

Oberauer, K. (2001). Removing irrelevant information from working memory: A cognitive aging study with the modified sternberg task. Journal of Experimental Psychology: Learning, Memory, and Cognition, 27, 948-957.

Niedenthal, P. \& Setterlund, M. (1994). Emotion congruence in perception. Personality and Social Psychology, 20(4), 401-4l1.

Uhlarik, J., Pringle, R., Jordan, K., \& Misceo, G. (1980). Size scaling in two dimensional pictorial arrays. Perception \& Psychophysics, 27(1), 60-70. 